Istituto di Genetica Medica «G. Mendel» (Roma)

Direttore Prof. L. Gedda

Dott. F. Sodani

\title{
Analisi della mortalità di alcune malattie ematologiche nella popolazione Italiana
}

Le prime statistiche ufficiali della mortalità, distinta per cause di morte, in Italia risalgono al i887. Pur tuttavia le classificazioni nosologiche che si sono succedute nel tempo, con conseguenti passaggi delle forme morbose in raggruppamenti diversi, rendono difficile individuare le singole malattie risalendo addietro per un congruo numero di anni.

Tra le malattie ematologiche quelle che sono individuabili per il più lungo numero di anni possibile sono, in ordine di tempo: emofilia e porpora (raggruppate) dal i887; anemia perniciosa dal I924; leucemia e m. di Hodgkin (raggruppate) dal I93 I. Attualmente in Italia è in uso la classificazione internazionale analitica approvata dalla convenzione del I948, adottata a partire dal I95I. Con questa classificazione le malattie del sangue vengono esaminate abbastanza dettagliatamente, ma le statistiche riguardano necessariamente un periodo di tempo molto breve, per cui si è preferito limitare l'analisi alle serie storiche che è possibile avere in base alle vecchie classificazioni. Utilizzando cifre pubblicate dall'Istituto Centrale di Statistica sono stati ricavati i dati che sono serviti per la costruzione delle rappresentazioni grafiche qui riportate.

Nella (fig. I) è raffigurata la mortalità per i tre gruppi di malattie suddette, su un milione di abitanti. Le cifre della mortalità, ottenute com'è noto dividendo il numero dei morti in ogni anno per la popolazione dello stesso anno, e moltiplicando il quoziente ottenuto per un milione, sono riportate sulla scala verticale, a sinistra. La scala orizzontale, in basso, reca i singoli anni, dal rgor al i955.

La linea spezzata inferiore (a tratto intero) rappresenta a partire dall'anno igo I la mortalità per emofilia, porpora ed altre affezioni emorragiche (diatesi emorragica, ipoprotrombinemia, malattia di Werlhoff etc.). 
Questo gruppo comprende, secondo la classificazione attuale, diverse entità nosologiche, le quali nelle vecchie classificazioni erano fuse sotto la denominazione di porpora primitiva, non essendo state ancora ben individuate dalla scienza medica. Questo gruppo mantiene costanti i suoi componenti per tutta la serie storica considerata, perciò pur essendo un gruppo misto è stato considerato ugualmente, perchè è l'unico per cui si possa risalire tanto addietro negli anni.

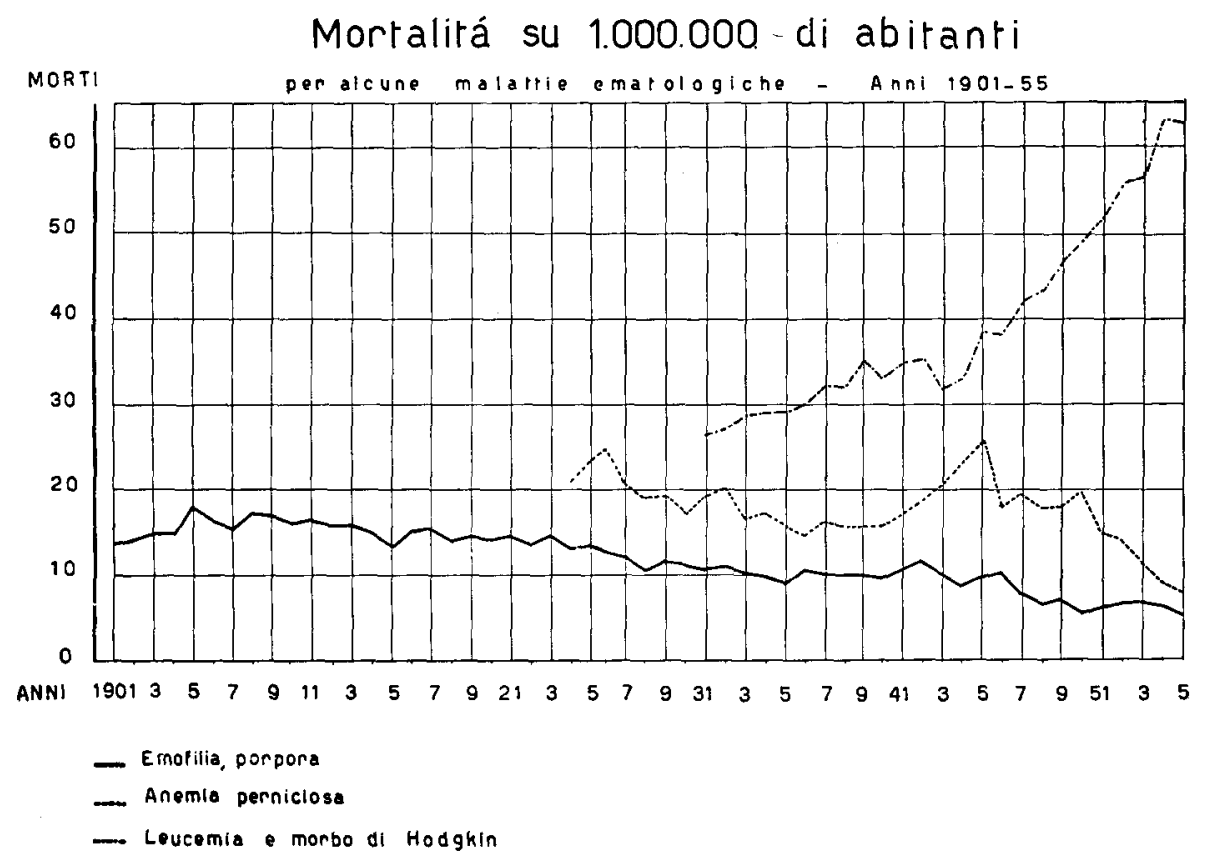

Fig. I

Si noti l'andamento lentamente decrescente, con una punta massima nel igo5 ( 7,8 morti su un milione di abitanti) ed una minima nel ig55 (5,2). Si noti ancora la tendenza della curva a salire negli anni della guerra e del dopoguerra (I94 I-I946).

Si noti una punta decrescente nel 1944, che potrebbe imputarsi ad una probabile dispersione di notizie causata dalla guerra che allora infieriva particolarmente in Italia.

La linea spezzata intermedia, a partire dall'anno r924, rappresenta la mortalità per anemia perniciosa e manifestazioni neuro-anemiche. Anche in essa, come nella precedente, si nota che l'andamento generale, pur essendo più irregolare, è decrescente. Una notevole irregolarità appare nel r940. Infatti da una quota stazionaria (stabilizzatasi intorno a I5 morti su un milione di abitanti, negli anni 1936-I940) la linea si innalza continuamente, partendo dal I94I, fino a raggiungere nel 1945 la quota massima di 25,2 . 
L'ipotesi più verosimile sulle cause di tale fenomeno potrebbe ricercarsi nella scarsa alimentazione e nella difficoltà di procurarsi gli estratti di fegato negli anni della guerra. Questa ipotesi sembra confermata dalla rapida caduta della mortalità nel i 946 a soli I7,9 morti su un milione di abitanti, e dal suo continuo movimento decrescente, dapprima lento, poi più rapido, per cui nel I955 scende a 7,6 .

Esaminando le statistiche inglesi degli ultimi anni si rileva il medesimo fenomeno (da 38 morti su un milione di abitanti nel 1946 si passa gradualmente a i9 nel i955).

Quindi si conclude che la mortalità per anemia perniciosa ha un andamento nettamente decrescente; un andamento pure decrescente ha la mortalità per affezioni emorragiche. $\hat{\mathbf{E}}$ utile confrontare $\mathrm{i}$ dati della mortalità con quelli della morbosità. Questo confronto è possibile solo per gli ultimi due anni della serie esaminata, usando $\mathrm{i}$ dati dell'Istituto Nazionale Assicurazione Malattia.

Si noti che le cifre pubblicate da questo Ente si riferiscono ai soli iscritti principali aventi diritto alla indennità di malattia, quindi ad un universo formato da popolazione adulta in età lavorativa, che non è perciò rappresentativo della intera popolazione italiana. Comunque il calcolo dei quozienti dà per l'emofilia, la porpora e le altre affezioni emorragiche un numero di 68,0 casi su un milione di assicurati nell'anno i954 e di 83,5 nell'anno i955. E notevole quindi la differenza con le cifre della mortalità che sono rispettivamente di 5,8 e di 5,2 morti su un milione di abitanti.

Per l'anemia perniciosa si ha un quoziente di morbosità di 92,5 nel 1954 e di I03, I nel I955, contro una mortalità di 8,8 e di 7,6 .

Questi dati sembrerebbero confermare che la decrescenza della mortalità sia attribuibile (almeno in parte) più al miglioramento dei presidi terapeutici che consentono così la guarigione, piuttosto che alla diminuzione del numero dei casi di malattia.

Sempre nella (fig. I) la linea spezzata superiore, la più alta, a partire dall'anno I93 I rappresenta la mortalità per leucemia e malattia di Hodgkin.

La curva mostra a colpo d'occhio un rapido andamento crescente, per cui la mortalità va da 26,3 morti su un milione di abitanti nel I93 I a 62,5 nel 1955 .

L'andamento continuamente crescente è disturbato da una lieve caduta nel I 940 e da una incisura piuttosto profonda negli anni I943 e I944, per cui da 35,o morti su un milione di abitanti nel r942 si scende a 3I,4 (nel I943) e 32,7 (nel I944),

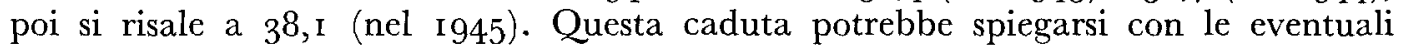
dispersioni di notizie e le difficoltà diagnostiche causate dai gravi disagi della guerra.

Nonostante questa irregolarità il rapido andamento crescente della curva è notevole. Anche le statistiche inglesi degli ultimi anni danno per la sola leucemia una mortalità rapidamente crescente che va da 35 morti su un milione di abitanti nel I 946 a $5^{\circ}$ nel r 955 .

Se si dovesse cercare una interpretazione delle cifre, una parte dell'aumento della mortalità per leucemia, risultante dalle statistiche, potrebbe attribuirsi al fatto che negli ultimi tempi si è avuto un continuo miglioramento dei mezzi diagnostici, per cui è più facile, per i medici che compilano le schede di morte (con le quali vengono fatte le statistiche) porre la diagnosi esatta; ma un'altra parte dell'aumento appare 
reale e concordante con l'aumentata mortalità per tutti i tumori maligni che si è verificata negli ultimi anni. Infatti in Italia nel I95 I la mortalità generale era di ro3o morti su centomila abitanti, la mortalità per tumori era di ben 122,2 morti su centomila abitanti e quella per malattie del sangue era di soli 4,3 morti su centomila abitanti (la leucemia nelle statistiche viene inclusa nel gruppo dei tumori).

Di fronte ad una così elevata mortalità per leucemia e morbo di Hodgkin negli ultimi anni, ${ }^{1}$ le cifre della morbosità (ricavate dai dati dell'I.N.A.M. per i quali valgono sempre le riserve fatte poc'anzi) danno una morbosità di $9 \mathrm{I}, 7$ (casi-malattia su un milione di assicurati) nel I954 e di 90,9 nel 1955 , cifre inferiori quindi a quelle della morbosità per anemia perniciosa che sono rispettivamente di 92,5 nel I954 e di Io3, I nel I 955 .

E interessante studiare la distribuzione per età delle malattie studiate nelle precedenti serie storiche. A tale scopo, per gli anni dal ig49 al I953, utilizzando la statistica dei morti distinti secondo le cause, il sesso e i gruppi di età, pubblicata dall'Istituto Centrale di Statistica, si è calcolata la media quinquennale (per ogni gruppo) allo scopo di perequare le divergenze dovute al caso; questa media è stata divisa per la popolazione dello stesso gruppo di età e sesso (rilevata dal censimento del 195I, anno centrale del quinquennio considerato) e rapportata a centomila abitanti. I

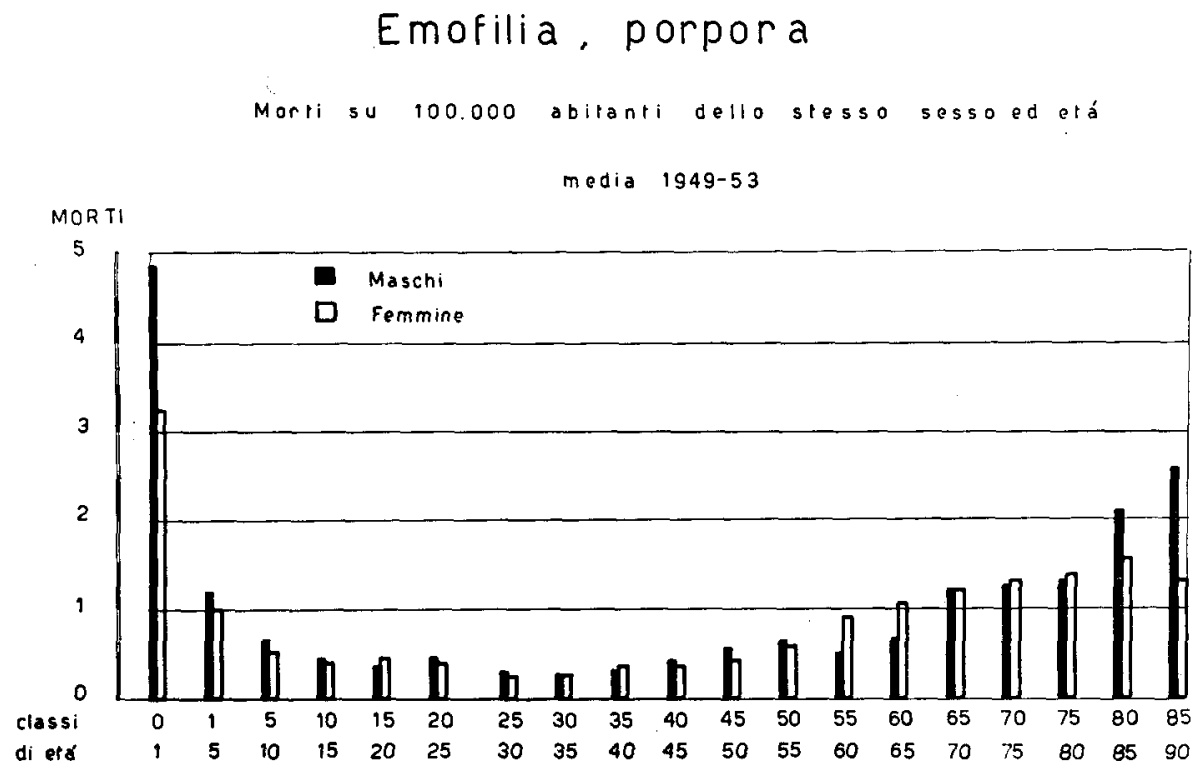

Fig. 2

162,9 morti su un milione di abitanti nel 1954 e 62,5 nel 1955. 
valori ottenuti sono stati tradotti graficamente, riportando sulla scala verticale, a sinistra, il numero dei morti; sulla scala orizzontale, in basso, le diverse classi di età.

Considerando l'istogramma riguardante l'emofilia la porpora e altre affezioni emorragiche (fig. 2) si nota l'elevato numero dei morti nel primo anno di età, nei confronti dei gruppi successivi. Nei primi cinque anni di età la mortalità maggiore è maschile; nelle classi successive la mortalità si equivale pressappoco tra i due sessi; nelle ultime classi si nota ancora la maggiore mortalità maschile.

Si potrebbe interpretare la elevata mortalità nel primo anno di età con una componente ereditaria, oltre che per i maschi (già scontata con l'emofilia) anche per le femmine.

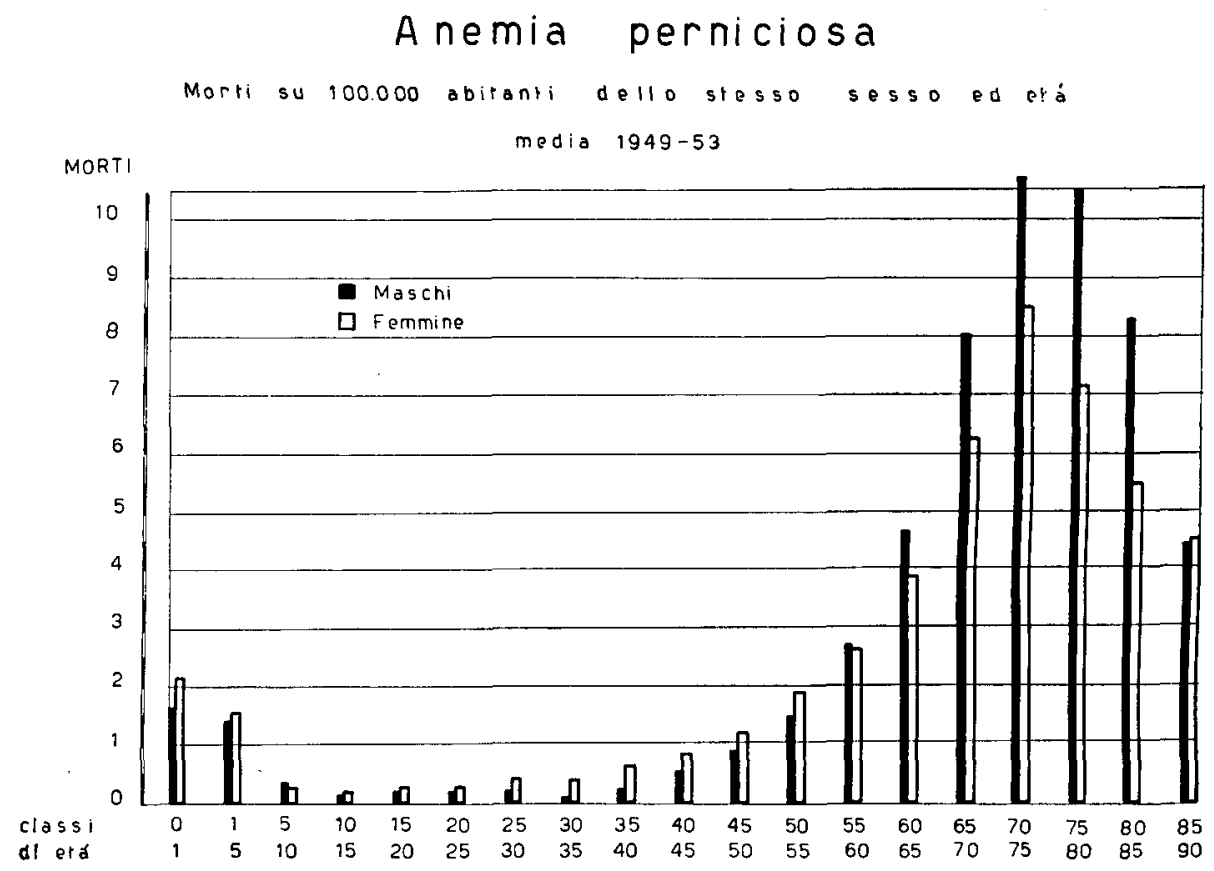

Fig. 3

Nel grafico riguardante l'anemia perniciosa (fig. 3) si nota che la mortalità, relativamente elevata nel primo e nel secondo gruppo di età (rispettivamente $\mathrm{r}$,6o per $\mathrm{i}$ maschi, 2, I 3 per le femmine e I,39 per i maschi, I,57 per le femmine) scende a livelli molto bassi $(0,2-0,3)$ nelle classi successive; si mantiene stazionaria fino a 40 anni di età, poi inizia la fase ascendente, raggiunge il massimo da 70 a 75 anni di età, inizia infine la fase discendente.

Un dato interessante è la lieve preponderanza del sesso femminile fino a 55 anni; successivamente la mortalità maschile prende nettamente il sopravvento. 
Nel grafico riguardante la leucemia (fig. 4) (considerata ora isolatamente dal morbo di Hodgkin) si nota che la mortalità è piuttosto elevata fin dalle prime due classi di età (intorno a 5 morti su centomila abitanti); nella classe successiva (da 5 a io anni di età) decresce; si stabilizza nelle classi comprese tra io e 40 anni di età (intorno a 2 morti su centomila abitanti); poi ascende progressivamente fino a raggiungere il massimo tra i maschi nella classe da 70 a 75 anni di età $(10,85)$ e tra le femmine nella classe da $6_{5}$ a 70 anni $(6,40)$.

E notevole la sempre costante e forte prevalenza della mortalità maschile.

Tutti e tre gli istogrammi esaminati ricordano la curva della mortalità generale, pur presentando ognuno caratteristiche particolari diverse.

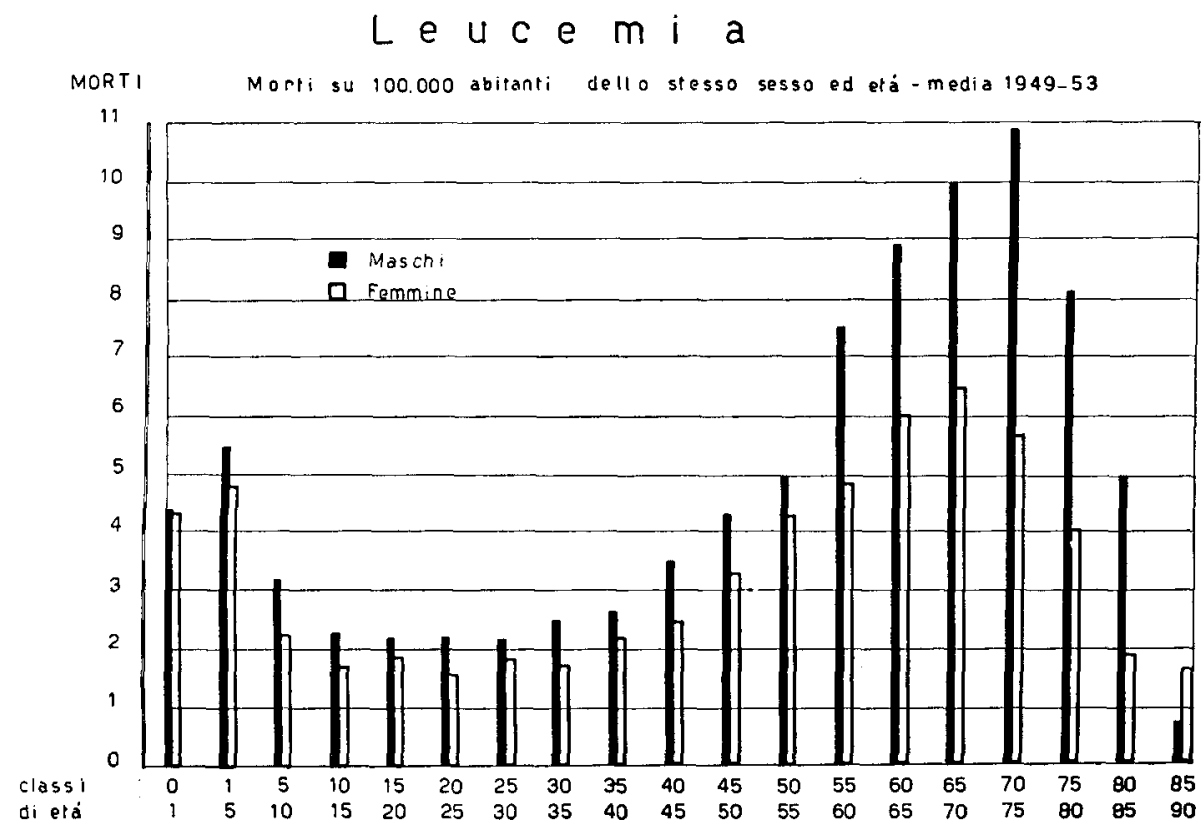

Fig. 4

In conclusione l'analisi statistica della mortalità per emofilia, porpora ed altre affezioni emorragiche; per anemia perniciosa; per leucemia e morbo di Hodgkin, mostra, nelle serie storiche, un andamento decrescente delle affezioni emorragiche e dell'anemia perniciosa e un andamento nettamente crescente della leucemia e morbo di Hodgkin.

L'analisi della mortalità distinta per età mostra, per tutte le forme morbose considerate, una mortalità più forte nella prima infanzia e nell'età avanzata; per la leucemia la mortalità maschile è nettamente maggiore di quella femminile. 


\section{Riassunto}

E stata esaminata la mortalità in Italia per i seguenti gruppi di cause: emofilia, porpora ed altre affezioni emorragiche;

anemia perniciosa;

leucemia e malattia di Hodgkin.

L'esame delle serie storiche mostra un andamento decrescente della mortalità per $\mathrm{i}$ primi due gruppi di cause e rapidamente crescente per il terzo gruppo.

L'esame della mortalità distinta per età e per sesso mostra nei tre gruppi di cause un'alta mortalità al di sotto dei 5 anni e al di sopra dei $5^{0}$ anni di età, pur con caratteristiche diverse da un gruppo all'altro.

La mortalità per leucemia è più alta nei maschi.

\section{RÉSUMÉ}

SUMMARY

On a examiné la mortalité en Italie pour les groupes de causes sous indiqués:

Hémophilie, purpura et autres affections hémorragiques;

Anémic pernicieuse;

Leucémie et maladie de Hodgkin.

L'examen des séries historiques montre une tendence décroissante de la mortalité pour les deux premiers groupes et rapidement croissante pour le troisième groupe.

L'examen de la mortalité, distingué selon l'âge et le sexe, montre que pour les trois groupes la mortalité est plus élevée au-dessous de cinq ans et au-dessus de cinquante ans, avec, cependant, des caractéristiques différentes d'un groupe à l'autre.

La mortalité pour la leucémie est plus élevée chez les hommes.
Death rates in Italy for the following groups of causes have been studied.

Haemophilia, purpura and other haemorrhagic conditions,

Pernicious anaemia,

Leukhaemia and Hodgkin's disease.

A study of the historical series shows a decreasing trend in death rates for the first two groups of causes and a rapidly increasing one for the third group. The study of death rates by age and sex shows, in the three groups of causes, a high death rate under 5 and over 50 years of age, with different characteristics from one group to another. Death rates for leukhaemia are higher in males than females.

\section{ZUSAMMENFASSUNG}

Es wird die Sterblichkeit in Italien für folgende Gruppen der Krankheiten geprüft: Hämophilie, Purpura und andere hämorragische Krankheiten;

Perniziöse Anämie;

Leukämie und Hodgkin'sche Krankheit.

Die Prüfung nach Jahren gesehen zeigt einen abnehmenden Gang der Sterblichkeit für die ersten zwei Gruppen der Krankheiten und einen rasch steigenden Gang für die dritte Gruppe der Krankheiten.

Die Prüfung der Sterblichkeit nach Alter und Geschlecht gesehen zeigt bei allen drei Gruppen der Krankheiten eine hochsteigende Sterblichkeit unter dem Alter von 5 Jahren und über s0 Jahren, aber mit verschiedener Charakteristik zwischen einzelnen Gruppen.

Die Sterblichkeit bei Leukämie ist Höher bei Männern als bei Frauen. 